\title{
Alcohol detoxification: a postal survey
}

\author{
Meena Agarwal, Keith Gaskell and Paul McArdle
}

\begin{abstract}
A survey of 92 in-patient alcohol dotoxification services revealed that they varied in thelr goals, organisation and treatment approaches. We argue that detoxlfication units and olcohol treatment units should increasingly take on the casesement and treatment role hitherto played by general poychlatilc wards in the management of the problem dinker. However, we also eugoest that the general poychiatilc wards should work closely with community acohol teams and more spectallst senvices in order to provide a comprehenstve service for these poitients.
\end{abstract}

In response to the Weiler report (1971), a number of detoxification centres were set up and studied (Hamilton et al, 1977; Arroyava et al, 1980). An evaluation of five examples of detoxification services (Detoxification Evaluation Project Research Team, 1985) indicated that they varied in their methods, staffing facilities, goals and clientele. Nevertheless certain key functions were agreed as desirable: accessibility, a treatment and rehabilitation programme which could respond to a range of needs, specialism among staff and continuity of aftercare with a network of community services (Royal College of Psychiatrists, 1986).

Although detoxification occurs in a variety of settings, hospitalisation may be required for severe dependence to provide careful observations and access to intensive medical and nursing care. The Lancaster district of north west England has provided a range of in-patient services for problem drinkers. In-patient detoxification on a psychiatric ward was replaced in November 1993 when a separate in-patient detoxification unit was opened after increased demand and dissatisfaction with psychiatric wards (Buckley, 1992). At that time, a lack of consensus and established guidelines on treatment programmes and policies became apparent.

The purpose of this survey was to gather information to form the basis of a clearer plan for current in-patient treatment and services. We surveyed various in-patient detoxification services and hypothesised that all services will have elements in common but differ in their organisation, treatment approaches and goals.

\section{The study}

An 11-item questionnaire (available on request from the authors) asking about alcohol detoxification (management of withdrawal from alcohol) was sent to a sample of $\mathbf{9 2}$ services providing in-patient detoxification in England and Wales. These included detoxification units (DUs), alcohol treatment units (ATUs) and general psychiatric wards (GPWs).

DUs are community based residential facilities offering specialist alcohol services, some of which are provided by voluntary organisations. They are run by multidisciplinary teams and medical cover is provided by local general practitioners. ATUs are hospital based specialist services and their primary function is to help alcohol dependent patients. Their staffing includes a specially trained and skilled multidisciplinary team. The GPWs provide an inpatient detoxification service as part of their overall work. They are not specialist alcohol services and are run by a multidisciplinary team.

Names and addresses of the DUs were collected from the Alcohol Services Directory (Alcohol Concern, 1991). Of the organisations listed under the category of residential facilities, only those that provided in-patient alcohol detoxification on the premises were included. Names and addresses of the ATUs were identified from the Alcohol Services Directory and Alcohol our Favourite Drug (Royal College of Psychiatrists, 1986). General psychiatric hospitals/units were identified from the directory of health authorities and the index to mental illness hospitals (Institute of Health Services Management, 1988).

The criterion for inclusion was provision of an in-patient alcohol detoxification service. All the ATUs ( $n=40)$ and DUs ( $n=12)$ then existing in England and Wales, and a random sample of $\mathbf{4 0}$ GPWs which fulfilled the inclusion criterion, were surveyed. Names of the responsible consultants/unit managers, were obtained via a telephone enquiry and letters were sent, requesting them to return the completed questionnaires in a stamped addressed envelope.

The questionnaire was designed to gather similar information from all units. However, the 
final question was open-ended asking for additional information regarding assessment/treatment (including specific guidelines/protocols) and unit profiles. In response, all DUs and $60 \%$ of the ATUs made specific comments and/or sent their detoxification protocols/guidelines along with their unit profiles. Information regarding referral patterns, client group, treatment regimes, aims and objectives and the range of services offered, was extracted. Similar information from GPWs was lacking.

\section{Findings}

Sixty-eight questionnaires were completed and returned, giving a response rate of $74 \%$. The percentage of return from each of the categories was as follows: DUs $58 \%(7 / 12)$, ATUs 73\% (29/ 40), and GPWs $80 \%$ (32/40) (see Tables 1 and 2).

\section{Detoxification units}

These units claimed to offer a "fast, direct access service". Significantly more units accept self and police referrals and referrals from agencies like probation services and Alcoholics Anonymous. In comparison to the ATUs and GPWs, the DUs were less selective. They reported making special provisions for 'homeless' and women alcoholics, drunken offenders and 'people in crisis'. Their exclusion criteria included severe psychiatric, medical, or drug problems. Their conditions of acceptance were that clients are sober on admission, violent behaviour is not tolerated and although clients may be accepted on ball or probation, they will not be admitted as a condition of a court order. Seventy-one per cent reported having none, or a few days, waiting period. They reported breathalysing clients and using a structured interview schedule for assessment. Like the ATUs, their aim was to provide a stress free, but a structured environment for detoxification.

Significantly more DUs used specific guidelines for detoxification. Almost half (43\%) of DUs reported using chlordiazepoxide as a first choice medication for detoxification, 29\% diazepam. $14 \%$ chlormethiazole and one DU used carbamazepine. While the trend concerning prescribed medication was similar to the ATUs and GPWs, none of the latter used carbamazepine as a first choice. The DUs, like the ATUs, carried out detoxification over a shorter period (36 hours5 days). They frequently used oral multivitamins and thiamine but did not report using additional medication except hypnotics, and referred patients on for treating complications. They reported 15 minutes observation and monitoring of withdrawal symptoms by nursing staff. Compared to the GPWs, significantly fewer DUs allowed unescorted leave during detoxification. Like the ATUs, they reported offering a range of services to meet clients' needs, a rehabilitation programme and a comprehensive aftercare plan.

\section{Alcohol treatment units}

These units also reported accepting referrals from a wide variety of sources but were more selective. They admitted primary alcoholics with severe dependence and in severe withdrawal states. They placed emphasis on patients' motivation to withdraw from alcohol. Their exclusion criteria were similar to DUs. They reported a waiting period of 2-4 weeks, due to service demand. They described a more specialised assessment using standardised measures of alcohol dependence and withdrawal symptoms.

Significantly more ATUs reported using detoxiflcation protocols adaptable to individual patients' requirements. These included standard/ modifled librium detoxification protocols, high/ low dose regimes, protocols for males/females of different ages/weights and for physically ill patients. Chlordiazepoxide was the commonest first choice medication for detoxification. Some units reported not using chlormethiazole because of its toxicity with alcohol. Carbamazepine was used if there was history of withdrawal seizures or epilepsy. The dose depended upon the severity of withdrawal symptoms. However more ATUs, compared to other types of units, used high initlal daily doses of chlordiazepoxide (100-300 mg) and reported using the Specific Severity Assessment Scale (SSA; available on request from the authors) for alcohol withdrawal symptoms to titrate dosage. ATUs reported using B vitamins if patients had malnutrition or peripheral neuropathy. Thiamine or Parbinex were used if there was a

Table 1. Accepted sources of referral

\begin{tabular}{llllll}
\hline Referral & $\begin{array}{l}\text { DUs }(n=7) \\
n(\%)\end{array}$ & $\begin{array}{l}\text { ATUs }(n=29) \\
n(\%)\end{array}$ & $\begin{array}{l}\text { GPWs (n=32) } \\
n(\%)\end{array}$ & $x^{2}($ d.f.=2) & $p$ \\
\hline Self & $6(86)$ & $24(83)$ & $15(47)$ & 8.72 & $<0.025$ \\
Pollce & $6(86)$ & $19(66)$ & $7(22)$ & 33.66 & $<0.0001$ \\
Social worker & $7(100)$ & $29(100)$ & $14(44)$ & 23.59 & $<0.0001$ \\
CPN & $7(100)$ & $28(97)$ & $17(53)$ & 11.59 & $<0.005$ \\
Other agencles & $7(100)$ & $29(100)$ & $17(53)$ & 13.23 & $<0.005$ \\
\hline
\end{tabular}


Table 2. Significant differences among different types of units

\begin{tabular}{|c|c|c|c|c|c|}
\hline Varicble & $\begin{array}{l}\text { DU: }(n=7) \\
n(\%)\end{array}$ & $\begin{array}{l}\text { ATUs }(n=29) \\
n(\%)\end{array}$ & $\begin{array}{l}\text { GPW/ (n=32) } \\
n(x)\end{array}$ & $x^{2}$ (d.f. $\left.=2\right)$ & $P$ \\
\hline $\begin{array}{l}\text { Walting period } \\
\text { over } 2 \text { weeks }\end{array}$ & 2 (29) & $17(59)$ & $12(38)$ & 8.76 & $<0.025$ \\
\hline $\begin{array}{l}\text { Nature of treatment } \\
\text { detoxification only } \\
\text { detoxification and rehabilitation }\end{array}$ & $\begin{array}{ll}2 & (29) \\
5 & (71)\end{array}$ & $\begin{array}{r}2(7) \\
27(93)\end{array}$ & $\begin{array}{r}25(78) \\
7(22)\end{array}$ & $\begin{array}{r}228.16 \\
69.86\end{array}$ & $\begin{array}{l}<0.0001 \\
<0.0001\end{array}$ \\
\hline $\begin{array}{l}\text { Assessment } \\
\text { standardised measures } \\
\text { Detoxification process }\end{array}$ & $0 \quad(0)$ & $8(28)$ & $3(9)$ & 8.46 & $<0.025$ \\
\hline $\begin{array}{l}\text { protocol } \\
\text { chlordiczepoxide over } 100 \mathrm{mg} \\
\text { Duration of detoxification }\end{array}$ & $\begin{array}{ll}4 & (57) \\
0 & (0)\end{array}$ & $\begin{array}{r}10(34) \\
9(31)\end{array}$ & $\begin{array}{l}0(0) \\
3(9)\end{array}$ & $\begin{array}{l}10.54 \\
12.37\end{array}$ & $\begin{array}{l}<0.01 \\
<0.005\end{array}$ \\
\hline $\begin{array}{l}\text { short } \\
\text { long }\end{array}$ & $\begin{array}{ll}3 & (43) \\
1 & (14)\end{array}$ & $\begin{array}{r}10(34) \\
8(28)\end{array}$ & $\begin{array}{r}4(13) \\
13(41)\end{array}$ & $\begin{array}{l}12.60 \\
16.48\end{array}$ & $\begin{array}{l}<0.005 \\
<0.001\end{array}$ \\
\hline $\begin{array}{l}\text { Aftercare } \\
\text { referral for rehabilltation } \\
\text { aftercare groups } \\
\text { counselling }\end{array}$ & $\begin{array}{l}7(100) \\
1(14) \\
4(57)\end{array}$ & $\begin{array}{r}19(66) \\
9(31) \\
26(90)\end{array}$ & $\begin{array}{r}12(38) \\
4(13) \\
25(78)\end{array}$ & $\begin{array}{r}11.22 \\
6.57 \\
6.92\end{array}$ & $\begin{array}{l}<0.005 \\
<0.05 \\
<0.05\end{array}$ \\
\hline $\begin{array}{l}\text { Unescorted leave } \\
\text { not allowed }\end{array}$ & 4 (57) & $21(72)$ & $13(41)$ & 6.60 & $<0.05$ \\
\hline
\end{tabular}

suspicion of Wernicke's encephalopathy. They prescribed anticonvulsants if patients had a history of epilepsy, withdrawal seizures or recent benzodiazepine use or if admitted in severe withdrawal. Seventeen per cent of the ATUs used carbamazepine as a first choice anticonvulsant; $17 \%$ used other medication as required, including atenolol for hypertension, haloperidol for psychosis and magnesium supplements. One unit used frusemide if withdrawal was unstable. They did not allow unescorted leave during detoxification for medical reasons. The length of stay was significantly longer in the ATUs than in GPWs.

\section{General psychiatric wards}

The GPWs accepted formal referrals but significantly fewer compared to DUs and ATUs accepted informal and police referrals. Nineteen per cent accepted admissions only if arranged by community alcohol teams. The GPWs were also selective and reported that suitability for admission depended upon the current level of dependence, previous delirium tremens or fits, social support, physical state and drug problems. Their waiting period depended on the demand for detoxification/general psychiatric beds. They rarely used standardised alcohol questionnaires for assessment.

Two-thirds of the GPWs reported a flexdble treatment regime depending on individual patient's requirements. One respondent used chlormethiazole for in-patients and diazepam for out-patients. Another prescribed chlordiazepoxdde for beer drinking and chlormethiazole for spirit drinking. One other respondent reported using a high potency intravenous multivitamin, Parbinex, as a main withdrawal cover treatment and chlormethiazole as an adjunct. Oxazepam was used if patients' hepatic functions were impaired. They used variable initial daily doses but compared to DUs and ATUs, GPWs prescribed the highest doses of diazepam (>80 mg) and chlormethiazole (>13 tablets).

In comparison to the DUs and ATUs, GPWs carried out detoxification over a longer period (>9 days). Thirty-eight per cent of the GPWs used vitamins, 9\% anticonvulsants and $12 \%$ other additional medication if required. Fiftynine per cent of the GPWs allowed unescorted leave at any stage of the treatment. The length of stay on a GPW was much shorter than in DUs or ATUs. GPWs did not offer a full range of services nor a comprehensive aftercare plan for alcohol dependent patients. Several respondents commented on the appropriateness of GPWs in this field.

\section{Comment}

Our findings confirm the hypothesis that all units share a similar function of providing inpatient detoxification under supervision but vary in the nature of their treatment regimes and goals. The community based DUs offer a rapid response, direct access and are less selecttve. The ATUs are highly specialised but have a longer waiting list and their use of very high doses of detoxification medication may reflect selective admissions of patients with severe withdrawal symptoms. The objectives of GPWs 


\section{ORIGINAL PAPERS}

include meeting the needs of general psychiatric patients. Their goal is 'drying out' people under supervision and not necessarily rehabilitation or the attainment of a new lifestyle. They may see continuity of care as important but have limited resources and detoxification alone may not be sufficient in the long term management of alcohol dependent patients. However, if working closely with community alcohol teams, these caveats do not fully apply.

DUs, ATUs and GPWs are not equivalent and serve different sections of the drinking population. DUs offer services to clients with alcohol related problems of intermediate severity while ATUs and GPWs assess and treat those with the most severe symptoms. GPWs do not claim special expertise and may feel uneasy dealing with alcohol dependent patients. Nevertheless, trends towards shorter waiting times for admission and for the highest doses of medication suggest they offer a rapidly accessible response to an ill group. This is clearly a valuable role, unlikely to be superseded without a major expansion in more specialist services. Nevertheless, to provide a comprehensive service for these patients, they should work closely with community alcohol teams and more specialist services.

\section{Acknowledgement}

We thank all respondents who participated in this survey.

\section{References}

ALCOHOL CONCERN (1991) Alcohol Services Directory. Ilkley: Owen Wells.

ARROYAVA, F., COOPER, S. E. \& HARRIS, A. D. (1980) The role of detoxification in alcoholism: three years' results from the Oxford Unit. Health Trends, 12, 36-38.

Buckiex, J. (1992) Alcohol Services in the North Western Reglon: a user's perspectlue. Manchester: North Western Reglonal Health Authority.

Detoxthication Evaluation Prosect Research Team (1985) Problem Drinking: Experiments in Detaxification. London: Bedford/NCVO.

HAMmTON, J. R., GRIFTtTHS M. A., RTISON, E. B., et al (1977) A detoxification unit for habitual drunken offenders. Health Bulletin, 35, 146-154.

InstTtute of HEalth SERVices Management (1988) The Hospitals and Health Services Year Book led N. W. Chaplin). London: IHSM.

Royal COLLEge of PSYchIATRISTs (1986) Aloohol our Favourtte Drug. London and New York: Tavistock.

WEnER, T. G. (1971) Habttual Drunken Offenders: Report of the Working Party. London: HMSO.

* Meena Agarwal, Senior Registrar in Child and Adolescent Psychiatry. Fleming Nuffield Unit for Children and Young People, Burdon Terrace, Jesmond, Newcastle upon Tyne NE2 3AE; Kelth Gaskell, Consultant in General Psychiatry, Ridge Lea Hospital, Quernmore Road, Lancaster LAI 3JR; and Paul McCardle, Consultant and Senior Lecturer in Child and Adolescent Psychiatry, Fleming Nuffield Unit for Children and Young People

"Correspondence 